\title{
Traduire
}

Une eutre perspective sur $r$ tatadciction

Revue française de la traduction

$221 \mid 2009$

Voies de l'interprétation

\section{Le traducteur juridique face à la différence}

\section{Malcolm Harvey}

\section{(2) OpenEdition}

Journals

Édition électronique

URL : http://journals.openedition.org/traduire/347

DOI : 10.4000/traduire.347

ISSN : 2272-9992

\section{Éditeur}

Société française des traducteurs

\section{Édition imprimée}

Date de publication : 15 décembre 2009

Pagination : 79-85

ISSN : 0395-773X

\section{Référence électronique}

Malcolm Harvey, «Le traducteur juridique face à la différence », Traduire [En ligne], 221 | 2009, mis en ligne le 12 novembre 2013, consulté le 25 novembre 2020. URL : http://journals.openedition.org/ traduire/347 ; DOI : https://doi.org/10.4000/traduire.347 


\section{Le traducteur juridique face à la différence}

\section{Malcolm Harvey}

Le traducteur juridique se heurte constamment à la différence. Rendre les notions d'une langue juridique par le biais d'une autre, c'est confronter deux systèmes, deux démarches, deux cultures juridiques. À la différence des sciences dites " exactes " (la physique, la chimie etc.), qui sont à vocation universelle, le droit se cantonne en grande partie dans les frontières nationales, chaque pays ayant élaboré son système de droit. Essayer de superposer deux cultures juridiques pour les besoins de la traduction met en évidence une incongruence : si certaines notions sont partagées, bien d'autres sont propres à chaque système.

À titre d'exemple, le droit français et le droit anglais contiennent un fonds commun dû aux convergences de l'histoire et, plus récemment, à l'émergence d'un système supranational, à savoir le droit communautaire. Mais à côté de ces points communs il existe des spécificités, à commencer par le fait que les deux systèmes appartiennent à des familles différentes. Le droit français fait partie de la famille romano-germanique, alors que le droit anglais a fondé la famille du common law, appelé aussi «le droit anglo-saxon ".

Cette différence d'appartenance a de nombreuses ramifications. D'une part, l'organisation de la justice n'est pas la même : le système français pratique une distinction, héritée du droit romain, entre le droit public et le droit privé, qui structure l'organisation des juridictions et des acteurs de la justice. Cette opposition existe aussi en droit anglais mais elle n'est pas fondamentale : la principale subdivision se fait entre le droit civil et le droit pénal(1).

D'autre part, les deux systèmes n'adoptent pas la même démarche. Le juriste français privilégie une approche déductive, partant de principes généraux qu'il applique ensuite au cas d'espèce. Cette approche reflète la prééminence accordée depuis la Révolution à la loi écrite. Au contraire, le juriste anglo-saxon préfère une démarche inductive, partant d'un cas

(1) La possibilité offerte en droit français d'associer les justices pénale et civile par la constitution de partie civile par la victime est étrangère au droit anglais. 
d'espèce pour dégager progressivement la règle de droit. Une telle démarche reflète le poids historique de la jurisprudence, qui accorde une plus grande place au juge. Même si la distinction a tendance à s'estomper(2), elle reste stucturante.

Une dernière distinction, sans doute la plus visible pour le néophyte, concerne la procédure pénale(3). La France a une procédure inquisitoire, c'est-à-dire fondée sur l'enquête : les affaires pénales sont instruites sous la direction du parquet ou, dans le cas de certains délits graves et des crimes, par cette figure emblématique qu'est le juge d'instruction(4). Une grande partie du travail se fait avant l'audience, le président étant habilité à prendre connaissance du dossier à l'avance. En revanche, l'Angleterre a une procédure accusatoire, où la recherche des preuves incombe aux parties. Ce système privilégie le débat oral et contradictoire : seuls les éléments de preuve évoqués à l'audience peuvent être pris en compte par le tribunal.

Ces divergences profondes et multiples ont pour résultat des termes spécifiques à chaque système juridique. Ceux-ci concernent notamment des institutions, les professions ou les concepts. Le problème qui se pose au traducteur est le suivant : comment trouver un équivalent pour un terme comme "Cour de cassation ", " notaire " ou " intime conviction " ? Cet article propose de passer en revue les différentes stratégies que peut adopter le traducteur, en pesant le pour et le contre de chacune d'entre elles. II soulignera par ailleurs le parti pris idéologique qui sous-tend le choix de telle ou telle technique, ainsi que la manière dont il nous renseigne sur le rôle du traducteur. Les exemples concernent la traduction du français vers l'anglais, mais les tendances dégagées peuvent s'appliquer à d'autres combinaisons de langues.

Les exemples cités proviennent de sites Internet monolingues en langue anglaise portant sur le droit français (voir la bibliographie). Les sources bilingues ou traduites ont été écartées, sauf quelques rares exceptions, pour privilégier la production spontanée. II n'est pas fait mention de la source de chaque exemple pour éviter de charger le texte, mais le lecteur pourra le cas échéant la retrouver en utilisant un moteur de recherche.

(2) La loi écrite, en particulier réglementaire, est devenue la principale source du droit en Angleterre ; a contrario la jurisprudence, même si elle n'est pas contraignante pour le juge français, est souvent utilisée comme argument par les parties.

(3) Les Français ont souvent une fausse idée de leur justice pénale en raison des séries télévisées américaines. Ainsi, le prévenu s'indigne parfois que son avocat ne mène pas une contre-enquête, alors que cette possibilité n'existe pas en droit français. De même, il exprime parfois son désaccord avec le président par la formule "Objection, votre Honneur! ", qui illustre les dangers d'une culture traduite.

(4) À I'heure où l'on écrit ces lignes, il est question de confier les enquêtes criminelles au parquet et de transformer le juge d'instruction en " juge de l'enquête et des libertés " exerçant un pouvoir de contrôle. Ces propositions sont formulées dans un rapport remis au Président de la République en septembre 2009 par le comité Léger. La procédure resterait inquisitoire mais accorderait en principe une plus grande place au débat contradictoire. 
L'étude du corpus met en évidence quatre principales techniques : l'équivalence culturelle, l'équivalence formelle, la traduction explicative et la transcription (accompagnée éventuellement d'une glose)(5). Il convient maintenant de les examiner à tour de rôle.

\section{L'équivalence culturelle}

Cette technique consiste à rechercher dans la langue cible un référent comparable, de par sa fonction, à celui de la langue source. Par exemple, le conseil des prud'hommes correspond peu ou prou en droit anglais à l'employment tribunal (autrefois appelé industrial tribunal). Cette technique est particulièrement prisée par les dictionnaires ou lexiques bilingues : ainsi, High Court et Crown Court sont donnés comme équivalents du tribunal de grande instance et de la cour d'assises respectivement.

L'avantage de cette technique est que le terme traduit est facile à comprendre (ou en tout cas à reconnaître) pour le néophyte : il a l'habitude d'entendre ces termes, même s'il ne sait pas exactement à quoi ils correspondent. Par ailleurs, l'équivalent crée une impression d'authenticité puisqu'il procède par analogie entre l'élément nouveau et une réalité déjà existante dans la culture cible.

Cette stratégie comporte néanmoins des dangers. Tout d'abord, dans une traduction à visée internationale, elle exclut certains lecteurs : ainsi, les équivalents proposés plus haut sont tous empruntés au système anglais et seront difficilement compréhensibles pour un anglophone non britannique. Le problème vient du fait qu'un terme spécifique à la culture source a été rendu par un terme spécifique à la culture cible.

II existe par ailleurs un risque d'imprécision. Par exemple, la traduction de " juge d'instruction " par examining magistrate est tellement fréquente qu'elle est pratiquement ancrée dans l'usage(6). Et pourtant, malgré leur similitude formelle ces deux termes recouvrent des réalités très différentes. L'examining magistrate vérifie si, à première vue, le dossier contient des preuves suffisantes pour juger l'affaire, alors que le juge d'instruction mène une enquête fouillée qui peut durer des mois, voire des années.

Dans certains cas l'équivalence culturelle peut prêter à confusion. Dans le doute, un bon test consiste à " rétrotraduire " le terme, c'est-à-dire que l'on retraduit le terme cible vers la langue source pour vérifier si l'on aboutit au terme d'origine. Ainsi, si l'on prend l'exemple de l'équivalence " cour d'assises " = Crown Court, la rétrotraduction de Crown Court peut générer

(5) Voir aussi M. Harvey, "A Beginner's Course in Legal Translation: the Case of Culture-bound Terms ", in Astti/Eti, La traduction juridique : histoire, théorie(s) et pratique, Bern/Genève, ASTTI/ETI, 2000, pp. 357-369.

(6) Une recherche sur www.google.com avec les mots-clés France "examining magistrate"a généré environ 15500 résultats. 
" cour d'assises " mais aussi " tribunal correctionnel ", dans la mesure où la Crown Court est compétente pour entendre certains délits relativement mineurs (qualifiés de triable either way offences) en plus des crimes.

De telles imprécisions peuvent sembler mineures aux yeux du néophyte mais elles sont significatives pour le juriste. C'est pourquoi l'équivalence culturelle peut convenir dans un texte adressé au grand public, mais elle est à manier avec précaution dans une traduction destinée au spécialiste.

Sur le plan idéologique, cette technique peut être taxée d'ethnocentrisme. Poussée à l'extrême, la volonté de tout expliquer par analogie avec une réalité déjà connue revient à gommer la différence, en créant l'impression d'une culture mondialisée et homogène. Cette approche n'est pas non plus valorisante pour le traducteur, car au lieu d'être un pont entre deux cultures il en efface l'une au profit de l'autre.

\section{L'équivalence formelle}

II s'agit d'une équivalence linguistique, autrement dit d'une traduction mot à mot (dans les limites de l'intelligibilité et de la grammaticalité). Ainsi, les termes " tribunal de police ", " cour d'assises " et " notaire " sont traduits par police court, assize court et notary.

Contrairement à l'équivalence culturelle, une telle traduction pourra être comprise indépendamment de la nationalité du lecteur (britannique, américain...) car elle ne fait pas appel à la culture cible. Par ailleurs, le risque d'ambiguité est moindre : si l'on applique le test de la rétrotraduction à assize court, la possibilité d'erreur est peu significative par rapport à l'équivalence culturelle Crown Court. C'est un facteur particulièrement important si le lecteur doit consulter des documents réalisés par différents traducteurs, car il assure de fait une certaine uniformité terminologique. A contrario, si chaque traducteur utilise un équivalent culturel différent pour désigner le même référent, cela crée une réelle confusion dans l'esprit du lecteur.

Cette technique a néanmoins plusieurs inconvénients. Le terme traduit peut paraître artificiel, voire obscur. Ainsi, les équivalents Assize Court et Court of Cassation, utilisés habituellement dans les traductions des arrêts de la Cour européenne des droits de l'homme, n'ont pas beaucoup de sens pour un anglophone non initié à cette terminologie transnationale. De manière plus extrême, le terme Council of State (utilisé comme équivalent de "Conseil d'État ") semble désigner un organe politique : le lecteur ne comprendra pas qu'il s'agit de la plus haute juridiction administrative en France. Le risque de calque n'étant jamais loin, l'équivalence formelle peut aboutir à une traduction obscure ou même absurde. Dans cette catégorie, la palme revient à Wikipedia qui, pour traduire " tribunal de grande instance ", propose Court of Large Instance... 
L'équivalence formelle ne devrait cependant pas être écartée d'emblée. Certes, une formule telle que Assize Court ou Court of Cassation risque de paraître étrange, voire incompréhensible, pour le lecteur "grand public ". En revanche le spécialiste, qui se soucie moins de questions esthétiques, appréciera l'absence d'ambiguité et le manque d'interférence avec la culture cible.

De manière plus générale, l'équivalence formelle se situe aux antipodes de l'équivalence culturelle car elle est résolument orientée vers la culture source : loin de gommer la différence, elle l'assume et dans certains cas l'accentue. C'est pour cela qu'elle se voit reprocher de " sentir la traduction " (ce qui est généralement synonyme de mauvaise traduction). Mais estce nécessairement un défaut ? Le traducteur doit-il donner l'impression que les cultures sont interchangeables, ou au contraire mettre en évidence les spécificités culturelles ? Par son étrangeté, ce procédé permet précisément d'attirer l'attention sur la différence au lieu de faire des amalgames trompeurs.

\section{La traduction descriptive}

Cette technique consiste en une explication qui est suffisamment concise pour fonctionner comme un terme autonome. Par exemple, " délit " = major offence, " crime " = serious crime, " juge d'instruction " = investigating judge.

Le principal avantage de ce type d'équivalence est sa transparence : l'absence de mots rares ou de calques lui permet d'être mieux compris par le lecteur, en particulier par le non-spécialiste. Par ailleurs, le terme est accessible pour l'ensemble de la communauté linguistique car il est composé de termes génériques, non spécifiques à une culture.

Une traduction descriptive peut toutefois manquer de précision. À titre d'exemple, le terme Criminal Court, proposé comme traduction de tribunal correctionnel, pourrait désigner n'importe quelle juridiction pénale (tribunal de police, tribunal correctionnel ou cour d'assises). De même, le terme limited liability company, utilisé pour traduire société à responsabilité limitée, peut correspondre à toute entreprise ayant la personnalité morale, par exemple une société anonyme. Ainsi, ce procédé est parfois insatisfaisant pour le spécialiste en raison du caractère nécessairement succinct de l'explication.

La traduction descriptive a l'avantage d'être accessible pour le lecteur néophyte, tout en évitant la " couleur locale " ou, au contraire, le poids écrasant de la culture cible. Elle reflète aussi une démarche plus active de la part du traducteur : au lieu de masquer la différence, il intervient discrètement pour la rendre lisible.

\section{La transcription}

Ce procédé consiste à reproduire le terme tel quel, en ajoutant éventuellement une glose lors de la première occurrence : par exemple, the Tribunal de Police (local criminal court) ou the 
Conseil d'État, the French highest administrative court. Le principal avantage de la transcription est qu'il n'y a aucun risque d'ambiguïté : même si le lecteur doit consulter des documents provenant de différents traducteurs, l'uniformité terminologique est assurée. C'est un critère important pour le spécialiste, ce qui explique en partie pourquoi ce procédé est de loin le plus utilisé dans les documents spécialisés consultés. Contrairement à la traduction descriptive, qui doit rester brève pour constituer un terme autonome, la glose peut être plus ou moins longue selon le degré de précision requis. Ainsi, pour expliquer le terme "tribunal d'instance ", on trouve la glose District Court mais aussi, dans une source plus spécialisée, lower district court of first instance (ce qui permet de différencier cette juridiction du tribunal de grande instance, expliqué par la glose higher district court of first instance).

En revanche, la transcription convient moins bien dans un texte destiné au profane, qui risque d'être rebuté par le recours fréquent à des termes étrangers (surtout s'il s'agit d'une langue qu'il ne connaît pas) et à des gloses qui interrompent la lecture.

Encore plus que l'équivalence formelle, la transcription affiche la différence. La langue source n'est plus voilée, mais au contraire mise en exergue. Le rôle du traducteur s'en trouve transformé : il sort de l'ombre pour intervenir directement dans le texte et pour guider le lecteur. Dans certains cas, il peut être amené à ajouter des notes en bas de page, voire des liens hypertexte pour proposer des explications plus fouillées(7). Ainsi, il assume pleinement son rôle de participant actif dans la communication, de "négociant en information(8) ".

Ces quatre techniques peuvent être différenciées grâce à la distinction, établie par Ladmiral, entre la traduction " sourcière " et la traduction " cibliste "(9). Si elles sont placées sur un continuum entre ces deux pôles, la transcription constitue le cas extrême de la traduction sourcière, suivie de l'équivalence formelle. L'équivalence culturelle se trouve à l'extrémité de la traduction cibliste, avant la traduction descriptive.

Le choix qui s'opère - consciemment ou inconsciemment - entre ces techniques reflète la manière dont le traducteur se situe dans le processus de la communication. S'il opte pour une solution " cibliste ", il essaie de se faire oublier, en créant l'illusion d'un " second original ». C'est le traducteur invisible, souvent présenté comme l'archétype du bon traducteur. Cachez ce traducteur que je ne saurais voir ! En revanche, s'il choisit une solution " sourcière ", il devient

(7) Cette approche a été largement exploitée lors de la rédaction de textes législatifs pour les anciennes républiques soviétiques, inspirés de documents russes ou anglais. Pendant la première phase de rédaction, les traducteurs ajoutaient de nombreuses notes pour expliquer les différences culturelles ou pour signaler d'éventuelles ambiguités ou erreurs. Voir C. Brown, "Riding the Waves of Fortune: Translating Legislation of the Successor Soviet Republics ", in M. Morris (éd), Translation and the Law, Amsterdam/Philadelphia, John Benjamins, 1995, pp. 67-83.

(8) G. Obenaus, "The Legal Translator as Information Broker ", in M. Morris (éd), pp. 247-259.

(9) J.-R. Ladmiral, "Sourciers et ciblistes ", Revue d'esthétique, n 12 (1986), pp. 33-42. 
acteur dans le processus de la communication : il confronte le lecteur à la différence et l'aide à s'y retrouver. Cette démarche peut être déconcertante de prime abord, mais le lecteur qui a besoin d'approfondir le sujet lui en saura gré. Du point de vue du traducteur, cette démarche " proactive " procure une plus grande satisfaction personnelle et peut aider à lui conférer un meilleur statut socioprofessionnel.

Malcolm.Harvey@univ-lyon2.fr

Malcolm Harvey est maître de conférences (anglais-LEA) à l'Université Lumière Lyon 2. Auteur d'une thèse de doctorat sur le langage juridique, il a également publié deux manuels de traduction : Méthode et pratique du thème anglais (en collaboration avec Michel Durand), Dunod, 1992 et L'Épreuve de thème anglais, Nathan, 2001.

\section{Bibliographie}

HARVEY Malcolm, "A Beginner's Course in Legal Translation: the Case of Culture-bound Terms", in Astti/Eti, La traduction juridique : histoire, théorie(s) et pratique, Berne/Genève, ASTTI/ETI, 2000, pp. 357-369. www.tradulex.org/Actes2000/harvey.pdf

LADMIRAL Jean-René, "Sourciers et ciblistes ", Revue d'esthétique, n 12 (1986), pp. 33-42.

MORRIS Marshall (éd), Translation and the Law, Amsterdam/Philadelphia, John Benjamins, 1995.

Exemples de sites Web consultés :

www.echr.coe.int/ECHR/EN/Header/Case-Law/HUDOC/HUDOC+database/

www.eurofound.eu.int/emire/FRANCE

http://europa.eu.int/smartapi/cgi/sga doc?smartapilcelexplus!prod!CELEXnumdoc\&numdoc $=6$ 1989J0066\&lg=en

www.financeinfrance.com/legal tax

www.frenchlaw.com/french litigation.htm

http://libcom.org/news/france-employment-law-ruled-be-breach-international-law-07072007 www.wgzavocats.com/articles/guidetoexport3a.html 